\title{
Article
}

\section{On Third-Order Differential Subordination and Superordination Properties of Analytic Functions Defined by a Generalized Operator}

\author{
Waggas Galib Atshan ${ }^{1, * \mathbb{D}}$, Rajaa Ali Hiress ${ }^{2}$ and Sahsene Altınkaya ${ }^{3} \mathbb{D}$

\footnotetext{
1 Department of Mathematics, College of Science, University of Al-Qadisiyah, Diwaniyah 58001, Iraq 2 Education of Al-Qadisiyah, Ministry of Education, Diwaniyah 58001, Iraq; waggashnd@gmail.com 3 Department of Mathematics, Beykent University, 34500 Istanbul, Turkey; sahsenealtinkaya@beykent.edu.tr * Correspondence: waggas.galib@qu.edu.iq
}

Citation: Atshan, W.G.; Hiress, R.A.; Altınkaya, S. On Third-Order Differential Subordination and Superordination Properties of Analytic Functions Defined by a Generalized Operator. Symmetry 2022, 14, 418. https://doi.org/ $10.3390 /$ sym 14020418

Academic Editors:

Alexander Zaslavski, Ioan Rașa, Sergei D. Odintsov and Juan Luis García Guirao

Received: 17 December 2021 Accepted: 13 January 2022

Published: 19 February 2022

Publisher's Note: MDPI stays neutral with regard to jurisdictional claims in published maps and institutional affiliations.

Copyright: () 2022 by the authors. Licensee MDPI, Basel, Switzerland. This article is an open access article distributed under the terms and conditions of the Creative Commons Attribution (CC BY) license (https:// creativecommons.org/licenses/by/ $4.0 /)$.

\begin{abstract}
In this current study, we aim to give some results for third-order differential subordination and superordination for analytic functions in $U=\{z \in \mathbb{C}:|z|<1\}$ involving the generalized operator $I_{\alpha, \beta}^{j} f$. The results are derived by investigating relevant classes of admissible functions. Some new results on differential subordination and superordination with some sandwich theorems are obtained. Moreover, several particular cases are also noted. The properties and results of the differential subordination are symmetry to the properties of the differential superordination to form the sandwich theorems.
\end{abstract}

Keywords: analytic function; subordination; superordination; sandwich result; dominant; subordinant

\section{Introduction}

Indicate by $\mathcal{H}=\mathcal{H}(U)$ the collection of analytic functions in the open unit disc $U$ that have the form:

$$
\begin{gathered}
\mathcal{H}[a, n]=\left\{f \in \mathcal{H}(U): f(z)=a+a_{n} z^{n}+a_{n+1} z^{n+1}+a_{n+2} z^{n+2}+\cdots\right\} \\
(a \in \mathbb{C}, n \in \mathbb{N}=\{1,2,3, \ldots\}),
\end{gathered}
$$

and indicate by $\mathcal{A}(n)$ the subclasses of $\mathcal{H}(U)$ comprising of functions

$$
f(z)=z+\sum_{k=n+1}^{\infty} a_{k} z^{k}, \quad(z \in U) .
$$

Note that $\mathcal{A}(1)=\mathcal{A}$. Further, let the functions $f_{1}$ and $f_{2}$ be in the class $\mathcal{H}(U)$. It is said that the function $f_{1}$ is subordinate to $f_{1}$ or $f_{2}$ is superordinate to $f_{1}$, if there exists a Schwarz function $\kappa(z)(\kappa(0)=0,|\kappa(z)|<1, z \in U)$ analytic in $U$ such that

$$
f_{1}(z)=f_{2}(\kappa(z))
$$

This subordination is indicated by $f_{1}(z) \prec f_{2}(z)$. Specifically, if the function $f_{2}$ is univalent in $U$, then we obtain (see [1])

$$
f_{1}(z) \prec f_{2}(z) \Leftrightarrow f_{1}(0)=f_{2}(0) \text { and } f_{1}(\mathrm{U}) \subset f_{2}(\mathrm{U}) \text {. }
$$

Now, we will recall the generalized operato $I_{\alpha, \beta}^{j}$ on $\mathcal{A}$ as below [2].

Suppose that $\beta \geq 0$ and $\alpha$ is a real number with $(\alpha+\beta)>0$. Then for $j \in \mathbb{N}_{0}=\mathbb{N} \cup\{0\}$ and $f \in \mathcal{A}$, the operator $I_{\alpha, \beta}^{j}$ is defined by 


$$
\begin{gathered}
I_{\alpha, \beta}^{0} f(z)=f(z) \\
I_{\alpha, \beta}^{1} f(z)=\frac{\alpha f(z)+\beta z f^{\prime}(z)}{\alpha+\beta} \\
\vdots \\
I_{\alpha, \beta}^{j} f(z)=I_{\alpha, \beta}\left(I_{\alpha, \beta}^{j-1} f(z)\right) .
\end{gathered}
$$

We see that $I_{\alpha, \beta}^{j}: \mathcal{A} \rightarrow \mathcal{A}$ is a linear operator and

$$
I_{\alpha, \beta}^{j} f(z)=z+\sum_{k=2}^{\infty}\left(\frac{\alpha+k \beta}{\alpha+\beta}\right)^{j} a_{k} z^{k}
$$

If $f \in \mathcal{A}(\mathrm{n})$, then the operator $I_{\alpha, \beta}^{j}$ is expressed by the infinite series:

$$
I_{\alpha, \beta}^{j} f(z)=z+\sum_{k=n+1}^{\infty}\left(\frac{\alpha+k \beta}{\alpha+\beta}\right)^{j} a_{k} z^{k}
$$

It is derived from (1) that

$$
\beta z\left(I_{\alpha, \beta}^{j} f(z)\right)^{\prime}=(\alpha+\beta) I_{\alpha, \beta}^{j+1} f(z)-\alpha I_{\alpha, \beta}^{j} f(z) .
$$

Further, for the particular values of $\alpha$ and $\beta$, Swamy [2] point out that the operator $I_{\alpha, \beta}^{j} f(z)$ reduces to various operators. Some of them are illustrated below:

- $\quad I_{\alpha, 0}^{j} f(z)=f(z)$;

- $\quad I_{1-\beta, \beta}^{j} f(z)=D_{\beta}^{j} f(z),(\beta \geq 0)$, known as Al-Oboudi differential operator [3];

- $\quad I_{\alpha, 1}^{j} f(z)=I_{\alpha}^{j} f(z),(\alpha>-1)$, investigated by Cho and Srivastava [4], Cho and Kim [5];

- $\quad I_{\gamma+1-\beta, \beta}^{j} f(z)=I_{\gamma, \beta}^{j} f(z),(\gamma>-1, \beta \geq 0)$, studied by Catas [6].

Antonino and Miller [7] (also [8,9]) have expanded the concept of second-order differential subordination and superordination in $U$ established by Miller and Mocanu $[1,10,11]$ to the third-order case. They derived features of functions $p$ that fulfill the third-order differential subordination:

$$
\left\{\psi\left(p(z), z p^{\prime}(z), z^{2} p^{\prime \prime}(z), z^{3} p^{\prime \prime \prime}(z) ; z\right): z \in U\right\} \subset \Omega,
$$

and also for third-order differential superordination:

$$
\Omega \subset\left\{\psi\left(p(z), z p^{\prime}(z), z^{2} p^{\prime \prime}(z), z^{3} p^{\prime \prime \prime}(z) ; z\right): z \in U\right\},
$$

where $\Omega$ is a set in $\mathbb{C}, p$ is an analytic function and $\psi: \mathbb{C}^{4} \times U \rightarrow \mathbb{C}$.

Recently, several authors studied some applications on the concept of second-order differential subordination and superordination and established some sandwich outcomes, like, (see $[12,13])$ and third-order for different classes (see $[8,9,14])$. For some interesting applications related to the differential subordination and superordination in other subjects of mathematics, we may refer to [15-17].

In order to demonstrate our outcomes, we shall give several definitions and theorems below.

Definition 1. (See [7]) Let $\psi: \mathbb{C}^{4} \times U \rightarrow \mathbb{C}$ and assume $h$ is univalent in $U$. If the function $p$ is analytic in $U$ and fulfills

$$
\psi\left(p(z), z p^{\prime}(z), z^{2} p^{\prime \prime}(z), z^{3} p^{\prime \prime \prime}(z) ; z\right) \prec h(z),
$$


then the function $p$ is named a solution of the differential subordination. A univalent function $q$ is named a dominant of the solution of the differential subordination if $p(z) \prec q(z)$ for all $p$ satisfying (2). A dominant $q(z)$ that fulfills $q^{\sim}(z) \prec q(z)$ for all dominants $q$ of (2) is named best dominant.

Definition 2. (See [9]) Let $\psi: \mathbb{C}^{4} \times U \rightarrow \mathbb{C}$ and assume $h$ is analytic in $U$. If the functions $p$,

$$
\psi\left(p(z), z p^{\prime}(z), z^{2} p^{\prime \prime}(z), z^{3} p^{\prime \prime \prime}(z) ; z\right)
$$

are univalent in $U$ and fulfill

$$
h(z) \prec \psi\left(p(z), z p^{\prime}(z), z^{2} p^{\prime \prime}(z), z^{3} p^{\prime \prime \prime}(z) ; z\right),
$$

then function $p$ is named a solution of the differential superordination. Further, an analytic function $q$ is named subordinant of the solutions of the differential superordination, or more simply a subordinant if $q(z) \prec p(z)$ for all $p$ satisfying (3). A univalent subordinant $q^{\sim}(z)$ that fulfills $q(z) \prec q^{\sim}(z)$ for all subordinants $q$ of (3) is named the best subordinant.

Definition 3. (See [7]) Indicate by $Q$. the set of all functions $q(z)$ that are analytic on $\bar{U} \backslash E(q)$, where,

$$
E(q)=\left\{\zeta: \zeta \in \partial U: \lim _{z \rightarrow \zeta} q(z)=\infty\right\},
$$

and are such that $q^{\prime}(\zeta) \neq 0$ for $\zeta \in \partial U \backslash E(q)$. Further, indicate by $Q(a)$ the subclass of $Q$ consisting of functions $q$ for which $q(0)=a$. Note that by $Q_{1}=Q(1)=\{q(z) \in Q: q(0)=1\}$.

Definition 4. (See [7]) Assume $\Omega$ be a set in $\mathbb{C}, q \in Q$ and $n \in \mathbb{N} \backslash\{1\}$. The class of admissible functions $\Psi_{n}[\Omega, q]$ consists of those functions $\psi: \mathbb{C}^{4} \times U \rightarrow \mathbb{C}$ that fulfill the following admissibility condition:

$$
\psi(r, s, t, u ; z) \notin \Omega,
$$

whenever

$$
\begin{aligned}
r=q(\zeta), \quad s=k \zeta q^{\prime}(\zeta), \operatorname{Re} & \left\{\frac{t}{s}+1\right\} \geq k \operatorname{Re}\left\{1+\frac{\zeta q^{\prime \prime}(\zeta)}{q^{\prime}(\zeta)}\right\}, \operatorname{Re}\left\{\frac{u}{s}\right\} \geq k^{2} \operatorname{Re}\left\{\frac{\zeta^{2} q^{\prime \prime \prime}(\zeta)}{q^{\prime}(\zeta)}\right\}, \\
& (z \in U, \zeta \in \partial U \backslash E(q) \text { and } k \geq n) .
\end{aligned}
$$

Definition 5. (See [9]) Let $\Omega$ be a set in $\mathbb{C}, q \in \mathcal{H}[a, n]$ with $q^{\prime}(z) \neq 0$ and $n \in \mathbb{N} \backslash\{1\}$. The class of admissible functions $\Psi_{n}^{\prime}[\Omega, q]$ consists of those functions $\psi: \mathbb{C}^{4} \times \bar{U} \rightarrow \mathbb{C}$ that satisfy the following admissibility condition:

$$
\psi(r, s, t, u ; z) \in \Omega,
$$

whenever

$$
\begin{gathered}
r=q(z), \quad s=\frac{z q^{\prime}(z)}{m}, \operatorname{Re}\left\{\frac{t}{s}+1\right\} \leq \frac{1}{m} \cdot \operatorname{Re}\left\{1+\frac{z q^{\prime \prime}(z)}{q^{\prime}(z)}\right\}, \operatorname{Re}\left\{\frac{u}{s}\right\} \leq \frac{1}{m^{2}} \operatorname{Re}\left\{\frac{z^{2} q^{\prime \prime \prime}(z)}{q^{\prime}(z)}\right\}, \\
(z \in U, \zeta \in \partial U m \geq n \geq 2) .
\end{gathered}
$$

Theorem 1. (See [7]) Assume $p \in \mathcal{H}[a, n](n \geq 2)$. Further, let $q \in Q(a)$ fulfill the conditions: 


$$
\operatorname{Re}\left\{\frac{\zeta q^{\prime \prime}(\zeta)}{q^{\prime}(\zeta)}\right\} \geq 0,\left|\frac{z p^{\prime}(z)}{q^{\prime}(\zeta)}\right| \leq k,(z \in U, \quad \zeta \in \partial U \backslash E(q) \text { and } k \geq n) .
$$

If $\Omega$ is a set in $\mathbb{C}, \psi \in \Psi_{n}[\Omega, q]$ and

$$
\psi\left(p(z), z p^{\prime}(z), z^{2} p^{\prime \prime}(z), z^{3} p^{\prime \prime \prime}(z) ; z\right) \in \Omega,
$$

then

$$
p(z) \prec q(z) \quad(z \in U) .
$$

Theorem 2. (See [9]) Let $\psi \in \Psi_{n}^{\prime}[\Omega, q]$. If $\psi\left(p(z), z p^{\prime}(z), z^{2} p^{\prime \prime}(z), z^{3} p^{\prime \prime \prime}(z) ; z\right)$ is univalent in $U, p \in Q(a)$ and $q \in \mathcal{H}[a, n]$ fulfill

$$
\operatorname{Re}\left\{\frac{\zeta q^{\prime \prime}(\zeta)}{q^{\prime}(\zeta)}\right\} \geq 0,\left|\frac{z p^{\prime}(z)}{q^{\prime}(\zeta)}\right| \leq m,(z \in U, \quad \zeta \in \partial U \text { and } m \geq n \geq 2),
$$

then

$$
\Omega \subset\left\{\psi\left(p(z), z p^{\prime}(z), z^{2} p^{\prime \prime}(z), z^{3} p^{\prime \prime \prime}(z) ; z\right): z \in U\right\},
$$

implies that

$$
q(z) \prec p(z) \quad(z \in U) .
$$

The current paper utilizes the techniques on the third-order differential subordination and superordination outcomes of Antonino and Miller [7], Ali et al. [18] and Tang et al. [9], respectively and different conditions (see $[19,20])$. Certain classes of admissible functions are investigated in this current paper, some properties of the third-order differential subordination and superordination for analytic functions in $U$ related to the operator $I_{\alpha, \beta}^{j} f$ are also mentioned.

\section{Third-Order Differential Subordination Properties}

This part includes third-order differential subordination properties are derived for analytic involving the generalized operator $I_{\alpha, \beta}^{j} f$.

Definition 6. Let $q \in Q_{0} \cap \mathcal{H}$ and $\Omega$ be a set in $C$. The class of admissible functions $\Phi_{I, 1}[\Omega, q]$ consists of those functions $\phi: \mathbb{C}^{4} \times U \rightarrow \mathbb{C}$ that fulfill the admissibility condition:

$$
\phi(u, v, x, y ; z) \notin \Omega \text {, }
$$

whenever

$$
\begin{gathered}
u=q(\zeta), v=\frac{k \zeta \beta q^{\prime}(\zeta)+\alpha q(\zeta)}{(\alpha+\beta)} \\
\operatorname{Re}\left\{\frac{(\alpha+\beta)^{2} x-2 \alpha(v \alpha+v \beta)+\alpha^{2} u}{\beta(v(\alpha+\beta)-\alpha u)}-1\right\} \geq k \operatorname{Re}\left\{\frac{\zeta q^{\prime \prime}(\zeta)}{q^{\prime}(\zeta)}+1\right\},
\end{gathered}
$$

and

$$
\begin{gathered}
\operatorname{Re}\left\{\frac{(\alpha+\beta)^{3} y-(3 \alpha+3 \beta)(\alpha+\beta)^{2} x+9 \alpha^{2} v \beta+6 \alpha v \beta^{2}-3 \alpha^{2} u \beta-\alpha^{3} u+3 \alpha^{3} v}{\beta^{2}(v(\alpha+\beta)-\alpha u)}+2\right\} \\
\geq k^{2} \operatorname{Re}\left\{\frac{\zeta^{2} q^{\prime \prime \prime}(\zeta)}{q^{\prime}(\zeta)}\right\}
\end{gathered}
$$


where $z \in U, \zeta \in \partial U \backslash E(q), \beta>0$ and $k \in \mathbb{N} \backslash\{1\}$.

Theorem 3. Let $\psi \in \Phi_{I, 1}[\Omega, q]$. If $f \in \mathcal{A}(n)$ and $q \in Q_{0} \cap \mathcal{H}$ fulfills the conditions

$$
\operatorname{Re}\left\{\frac{\zeta q^{\prime \prime}(\zeta)}{q^{\prime}(\zeta)}\right\} \geq 0, \quad\left|(\alpha+\beta) I_{\alpha, \beta}^{j+1} f(z)-\alpha I_{\alpha, \beta}^{j} f(z)\right| \leq k \beta\left|q^{\prime}(\zeta)\right|
$$

and

$$
\left\{\phi\left(I_{\alpha, \beta}^{j} f(z), I_{\alpha, \beta}^{j+1} f(z), I_{\alpha, \beta}^{j+2} f(z), I_{\alpha, \beta}^{j+3} f(z) ; z\right): z \in U\right\} \subset \Omega,
$$

then

$$
I_{\alpha, \beta}^{j} f(z) \prec q(z) \quad(z \in U, \quad \zeta \in \partial U \backslash E(q), \quad \beta>0 \text { and } k \in \mathbb{N} \backslash\{1\})
$$

Proof. Let us put

$$
w(z)=I_{\alpha, \beta}^{j} f(z) .
$$

By differentiating (6) with respect to $z$ and from (1), we find

$$
I_{\alpha, \beta}^{j+1} f(z)=\frac{\beta z w^{\prime}(z)+\alpha w(z)}{\alpha+\beta} .
$$

Further computations give

$$
I_{\alpha, \beta}^{j+2} f(z)=\frac{\beta^{2} z^{2} w^{\prime \prime}(z)+\left(\beta^{2}+2 \alpha \beta\right) z w^{\prime}(z)+\alpha^{2} w(z)}{(\alpha+\beta)^{2}}
$$

and

$$
I_{\alpha, \beta}^{j+3} f(z)=\frac{\beta^{3} z^{3} w^{\prime \prime \prime}(z)+\left(3 \alpha \beta^{2}+3 \beta^{3}\right) z^{2} w^{\prime \prime}(z)+\left(3 \alpha^{2} \beta+3 \alpha \beta^{2}+\beta^{3}\right) z w^{\prime}(z)+\alpha^{3} w(z)}{(\alpha+\beta)^{3}} .
$$

Now, we will establish a transformation from $\mathbb{C}^{4}$ to $\mathbb{C}$ by

$$
u(r, s, t, e)=r, \quad v(r, s, t, e)=\frac{\beta s+\alpha r}{(\alpha+\beta)}, \quad x(r, s, t, e)=\frac{\beta^{2} t+\left(\beta^{2}+2 \alpha \beta\right) s+\alpha^{2} r}{(\alpha+\beta)^{2}}
$$

and

$$
y(r, s, t, e)=\frac{\beta^{3} e+\left(3 \alpha \beta^{2}+3 \beta^{3}\right) t+\left(3 \alpha^{2} \beta+3 \alpha \beta^{2}+\beta^{3}\right) s+\alpha^{3} r}{(\alpha+\beta)^{3}} .
$$

Next, suppose

$$
\begin{gathered}
\psi(r, s, t, e ; z)=\phi(u, v, x, y ; z)=\phi\left(r, \frac{\beta s+\alpha r}{(\alpha+\beta)}, \frac{\beta^{2} t+\left(\beta^{2}+2 \alpha \beta\right) s+\alpha^{2} r}{(\alpha+\beta)^{2}},\right. \\
\left.\frac{\beta^{3} e+\left(3 \alpha \beta^{2}+3 \beta^{3}\right) t+\left(3 \alpha^{2} \beta+3 \alpha \beta^{2}+\beta^{3}\right) s+\alpha^{3} r}{(\alpha+\beta)^{3}} ; z\right) .
\end{gathered}
$$

It follows from (9) and Theorem 1 that

$$
\psi\left(w(z), z w^{\prime}(z), z^{2} w^{\prime \prime}(z), z^{3} w^{\prime \prime \prime}(z) ; z\right)=\phi\left(I_{\alpha, \beta}^{j} f(z), I_{\alpha, \beta}^{j+1} f(z), I_{\alpha, \beta}^{j+2} f(z), I_{\alpha, \beta}^{j+3} f(z) ; z\right) .
$$


Hence, the inclusion (5) leads to

$$
\psi\left(w(z), z w^{\prime}(z), z^{2} w^{\prime \prime}(z), z^{3} w^{\prime \prime \prime}(z) ; z\right) \subset \Omega .
$$

Moreover, in view of (7) and (8), we get

$$
\frac{t}{s}+1=\frac{(\alpha+\beta)^{2} x-2 \alpha(v \alpha+v \beta)+\alpha^{2} u}{\beta(v(\alpha+\beta)-\alpha u)}-1,
$$

and

$$
\frac{e}{s}=\frac{(\alpha+\beta)^{3} y-(3 \alpha+3 \beta)(\alpha+\beta)^{2} x+9 \alpha^{2} v \beta+6 \alpha v \beta^{2}-3 \alpha^{2} u \beta-\alpha^{3} u+3 \alpha^{3} v}{\beta^{2}(v(\alpha+\beta)-\alpha u)}+2 .
$$

Therefore, the admissibility condition in Definition 6 for $\phi \in \Phi_{\mathrm{I}, 1}[\Omega, q]$ is equivalent to the condition for $\psi \in \Phi_{2}[\Omega, q]$ as given in Definition 4 for $n=2$. Hence, by making use of (4) and applying Theorem 1 , we see that

$$
I_{\alpha, \beta}^{j} f(z) \prec q(z) .
$$

The next outcome is a direct conclusion of Theorem 3.

Theorem 4. Let $\phi \in \Phi_{I, 1}[h, q]$. If the functions $f \in \mathcal{A}(n)$ and $q \in Q_{0}$ fulfill the following conditions

$$
\operatorname{Re}\left\{\frac{\zeta q^{\prime \prime}(\zeta)}{q^{\prime}(\zeta)}\right\} \geq 0, \quad\left|(\alpha+\beta) I_{\alpha, \beta}^{j+1} f(z)-\alpha I_{\alpha, \beta}^{j} f(z)\right| \leq k \beta\left|q^{\prime}(\zeta)\right|
$$

and

$$
\left.\phi\left\{I_{\alpha, \beta}^{j} f(z), I_{\alpha, \beta}^{j+1} f(z), I_{\alpha, \beta}^{j+2} f(z), I_{\alpha, \beta}^{j+3} f(z) ; z\right): z \in U\right\} \prec h(z)
$$

then

$$
I_{\alpha, \beta}^{j} f(z) \prec q(z) .
$$

Proof. It is clear that by using Theorem 3, we arrive at the desired outcome.

The next corollaries are extensions of Theorem 3 to the case where the behavior of $q(z)$ on $\partial U$ is not known.

Corollary 1. Assume $\Omega \subset \mathbb{C}$ and $q(z)$ is univalent in $U$ with $q(0)=1, \phi \in \Phi_{I, 1}\left[\Omega, q_{\rho}\right]$ for some $\rho \in(0,1)$ where $q_{\rho}(z)=q(\rho z)$. If $f(z) \in \mathcal{A}(n)$ and $q_{\rho} \in Q_{0}$ fulfill

$$
\operatorname{Re}\left\{\frac{\zeta q_{\rho}^{\prime \prime}(\zeta)}{q_{\rho}^{\prime}(\zeta)}\right\} \geq 0, \quad\left|(\alpha+\beta) I_{\alpha, \beta}^{j+1} f(z)-\alpha I_{\alpha, \beta}^{j} f(z)\right| \leq k \beta\left|q_{\rho}^{\prime}(\zeta)\right|
$$

and

$$
\left.\phi\left\{I_{\alpha, \beta}^{j} f(z), I_{\alpha, \beta}^{j+1} f(z), I_{\alpha, \beta}^{j+2} f(z), I_{\alpha, \beta}^{j+3} f(z) ; z\right): z \in U\right\} \in \Omega,
$$

then

$$
I_{\alpha, \beta}^{j} f(z) \prec q_{\rho}(z)
$$

Proof. Theorem 3 yields $I_{\alpha, \beta}^{j} f(z) \prec q(z)$. The outcome is now deduced from $q_{\rho}(z) \prec q(z)$. 
Corollary 2. Let $\Omega \subset \mathbb{C}$ and suppose that $q(z)$ is univalent in $U$ with $q(0)=1, \phi \in \Phi_{I, 1}\left[\Omega, q_{\rho}\right]$ for some $\rho \in(0,1)$, where $q_{\rho}(z)=q(\rho z)$. If $f(z) \in \mathcal{A}(n)$ and $q_{\rho} \in Q_{0}$ fulfill

$$
\operatorname{Re}\left\{\frac{\zeta q_{\rho}^{\prime \prime}(\zeta)}{q_{\rho}^{\prime}(\zeta)}\right\} \geq 0, \quad\left|(\alpha+\beta) I_{\alpha, \beta}^{j+1} f(z)-\alpha I_{\alpha, \beta}^{j} f(z)\right| \leq k \beta\left|q_{\rho}^{\prime}(\zeta)\right|,
$$

and

$$
\phi\left(I_{\alpha, \beta}^{j} f(z), I_{\alpha, \beta}^{j+1} f(z), I_{\alpha, \beta}^{j+2} f(z), I_{\alpha, \beta}^{j+3} f(z) ; z\right) \prec h(z),
$$

then

$$
I_{\alpha, \beta}^{j} f(z) \prec q(z) .
$$

Proof. The outcome is similar to the proof of ([17], Theorem 2.3d, p. 30) and is therefore omitted.

Theorem 5. Let $\psi: \mathbb{C}^{4} \times U \rightarrow \mathbb{C}, h$ be univalent function in $U$ and $\psi$ be given by (9). Assume

$$
\psi\left(q(z), z q^{\prime}(z), z^{2} q^{\prime \prime}(z), z^{3} q^{\prime \prime \prime}(z) ; z\right)=h(z),
$$

has a solution $q(z) \in Q_{0} \cap \mathcal{H}$. If the function $f \in \mathcal{A}(n)$ fulfill (4) and

$$
\phi\left(I_{\alpha, \beta}^{j} f(z), I_{\alpha, \beta}^{j+1} f(z), I_{\alpha, \beta}^{j+2} f(z), I_{\alpha, \beta}^{j+3} f(z) ; z\right), \beta>0
$$

is analytic in $U$, then (11) implies that

$$
I_{\alpha, \beta}^{j} f(z) \prec q(z),
$$

and $q(z)$ is the best dominant.

Proof. From Theorem 3, we find that $q(z)$ is a dominant (11) since $q(z)$ fulfills

$$
\psi\left(q(z), z q^{\prime}(z), z^{2} q^{\prime \prime}(z), z^{3} q^{\prime \prime \prime}(z) ; z\right)=h(z),
$$

it is also a solution of the above differential equation and therefore $q(z)$ will be dominated by all dominants. Hence, $q(z)$ is the best dominant.

According to Definition 6 and for $q(z)=M z,(M>0)$, the class of admissible functions $\Phi_{\mathrm{I}, 1}[\Omega, q]=\Phi_{\mathrm{I}, 1}[\Omega, \mathcal{M}]$ is expressed below.

Definition 7. The class of admissible functions $\Phi_{I, 1}[\Omega, \mathcal{M}]$ consists of those functions $\psi: \mathbb{C}^{4} \times U \rightarrow \mathbb{C}$ such that

$$
\psi\left(\mathcal{M} e^{i \theta}, \frac{(\alpha+\beta k) \mathcal{M} e^{i \theta}}{(\alpha+\beta)}, \frac{\beta^{2} L+\left(\left(\beta^{2}+2 \alpha \beta\right) k+\alpha^{2}\right) \mathcal{M} e^{i \theta}}{(\alpha+\beta)^{2}}, \frac{\beta^{3} N+\left(3 \alpha \beta^{2}+3 \beta^{3}\right) L+\left(\left(3 \alpha^{2} \beta+3 \alpha \beta^{2}+\beta^{3}\right) k+\alpha^{3}\right) \mathcal{M} e^{i \theta}}{(\alpha+\beta)^{3}} ; z\right) \notin \Omega,
$$

where $z \in U, \beta>0, \operatorname{Re}\left(L e^{-i \theta}\right) \geq(k-1) k \mathcal{M}$, and for all $\theta \in \mathbb{R}$ and $k \in \mathbb{N} \backslash\{1\}$.

Corollary 3. Let $\psi \in \Phi_{I, 1}[\Omega, \mathcal{M}]$. If the function $f \in \mathcal{A}(n)$ fulfills the conditions:

$$
\left|(\alpha+\beta) I_{\alpha, \beta}^{j+1} f(z)-\alpha I_{\alpha, \beta}^{j} f(z)\right| \leq k \beta \mathcal{M},
$$

and

$$
\phi\left(I_{\alpha, \beta}^{j} f(z), I_{\alpha, \beta}^{j+1} f(z), I_{\alpha, \beta}^{j+2} f(z), I_{\alpha, \beta}^{j+3} f(z) ; z\right) \in \Omega,
$$


then

$$
\left|I_{\alpha, \beta}^{j} f(z)\right|<\mathcal{M}
$$

If $\Omega=q(U)=\{w:|w|<\mathcal{M}(\mathcal{M}>0)\}$, then the class $\Phi_{I, 1}[\mathcal{M}]$ is represented by $\Phi_{I, 1}[\Omega, \mathcal{M}]$.

Corollary 4. Let $\psi \in \Phi_{I, 1}[\mathcal{M}]$. If the function $f \in \mathcal{A}(n)$ fulfills

$$
\left|(\alpha+\beta) I_{\alpha, \beta}^{j+1} f(z)-\alpha I_{\alpha, \beta}^{j} f(z)\right| \leq k \beta \mathcal{M},
$$

and

$$
\left|\psi\left(I_{\alpha, \beta}^{j} f(z), I_{\alpha, \beta}^{j+1} f(z), I_{\alpha, \beta}^{j+2} f(z), I_{\alpha, \beta}^{j+3} f(z) ; z\right)\right|<\mathcal{M}
$$

and

$$
\phi\left(I_{\alpha, \beta}^{j} f(z), I_{\alpha, \beta}^{j+1} f(z), I_{\alpha, \beta}^{j+2} f(z), I_{\alpha, \beta}^{j+3} f(z) ; z\right) \in \Omega
$$

then

$$
\left|I_{\alpha, \beta}^{j} f(z)\right|<\mathcal{M}
$$

Corollary 5. Let $M>0, k \in \mathbb{N} \backslash\{1\}, \beta>0$ and $(\alpha+\beta)>0$. If the function $f \in \mathcal{A}(n)$. fulfills the following conditions:

$$
\left|I_{\alpha, \beta}^{j+2} f(z)-I_{\alpha, \beta}^{j+1} f(z)\right|<\frac{2 \beta^{2} \mathcal{M}+2 \alpha \beta \mathcal{M}}{(\alpha+\beta)^{2}},
$$

then

$$
\left|I_{\alpha, \beta}^{j} f(z)\right|<\mathcal{M}
$$

Proof. We put $\phi(u, v, x, y ; z)=x-v$. According to Corollary 3 with $\Omega=h(U)$ and $h(z)=\frac{2 \beta^{2} \mathcal{M}+2 \alpha \beta \mathcal{M}}{(\alpha+\beta)^{2}} z,(z \in U)$, we shall present that $\psi \in \Phi_{\mathrm{I}, 1}[\Omega, \mathcal{M}]$. Since

$$
\begin{gathered}
\phi\left(\mathcal{M} e^{i \theta}, \frac{(\alpha+\beta k) \mathcal{M} e^{i \theta}}{(\alpha+\beta)}, \frac{\beta^{2} L+\left(\left(\beta^{2}+2 \alpha \beta\right) k+\alpha^{2}\right) \mathcal{M} e^{i \theta}}{(\alpha+\beta)^{2}}\right. \\
\left.\frac{\beta^{3} N+\left(3 \alpha \beta^{2}+3 \beta^{3}\right) L+\left(\left(3 \alpha^{2} \beta+3 \alpha \beta^{2}+\beta^{3}\right) k+\alpha^{3}\right) \mathcal{M} e^{i \theta}}{(\alpha+\beta)^{3}}\right) ; z \\
=\left|\frac{\beta^{2} L+\alpha \beta k \mathcal{M} e^{i \theta}}{(\alpha+\beta)^{2}}\right| \\
=\left|\frac{\beta^{2} L e^{-i \theta}+\alpha \beta k \mathcal{M}}{(\alpha+\beta)^{2} e^{-i \theta}}\right| \\
\geq \frac{\beta^{2} \operatorname{Re}\left(L e^{-i \theta}\right)+k|\alpha \beta| \mathcal{M}}{(\alpha+\beta)^{2}} \\
\geq \frac{2 \beta^{2} \mathcal{M}+2 \alpha \beta \mathcal{M}}{(\alpha+\beta)^{2}}
\end{gathered}
$$

the proof is completed.

Now, we establish the next admissible class. 
Definition 8. Assume $q \in Q_{1} \cap \mathcal{H}$ and $\Omega$ is a set in $\mathbb{C}$. The class of admissible functions $\Phi_{I, 2}[\Omega, q]$ consists of those functions $\phi: \mathbb{C}^{4} \times U \rightarrow \mathbb{C}$ that fulfill the admissibility conditions:

$$
\phi(u, v, x, y ; z) \notin \Omega \text {, }
$$

whenever

$$
\begin{aligned}
& u=q(\zeta), \quad v=\frac{k \zeta \beta q^{\prime}(\zeta)+(\alpha+\beta) q(\zeta)}{(\alpha+\beta)}, \\
& \operatorname{Re}\left\{\frac{(\alpha+\beta)^{2} x-\beta^{2} u+\alpha^{2}(u-2 v)-2 \alpha \beta v}{\beta((\alpha+\beta) v-(\alpha+\beta) u)}-2\right\} \geq k \operatorname{Re}\left\{\frac{\zeta q^{\prime \prime}(\zeta)}{q^{\prime}(\zeta)}+1\right\}, \\
& \operatorname{Re}\left\{\frac{(\alpha+\beta)^{3} y-(3 \alpha+6 \beta)(\alpha+\beta)^{2} x+15 \beta \alpha^{2} v+12 \alpha \beta^{2} v+3 \alpha^{3} v-\alpha^{3} u-}{6 \alpha \beta u^{3}+5 \beta^{3} u}+11\right\} \geq k^{2} \operatorname{Re}\left\{\frac{\zeta^{2} q^{\prime \prime \prime}(\zeta)}{q^{\prime}(\zeta)}\right\},
\end{aligned}
$$

where $k \in \mathbb{N} \backslash\{1\}, \beta>0, \zeta \in \partial U \backslash E(q)$ and $z \in U$.

Theorem 6. Let $\psi \in \Phi_{I, 2}[\Omega, q]$. If the function $f \in \mathcal{A}(n)$ and $q \in Q_{1} \cap \mathcal{H}$ fulfills the following conditions

$$
\operatorname{Re}\left\{\frac{\zeta q^{\prime \prime}(\zeta)}{q^{\prime}(\zeta)}\right\} \geq 0, \quad\left|(\alpha+\beta)\left(\frac{I_{\alpha, \beta}^{j+1} f(z)-I_{\alpha, \beta}^{j} f(z)}{z}\right)\right| \leq k \beta\left|q^{\prime}(\zeta)\right|
$$

and

$$
\left\{\phi\left(\frac{I_{\alpha, \beta}^{j} f(z)}{z}, \frac{I_{\alpha, \beta}^{j+1} f(z)}{z}, \frac{I_{\alpha, \beta}^{j+2} f(z)}{z}, \frac{I_{\alpha, \beta}^{j+3} f(z)}{z} ; z\right): z \in U\right\} \subset \Omega,
$$

then

$$
\frac{I_{\alpha, \beta}^{j} f(z)}{z} \prec q(z) .
$$

Proof. Let put

$$
w(z)=\frac{I_{\alpha, \beta}^{j} f(z)}{z} .
$$

Then, by differentiating (14) with respect to $z$ and from (1), we find that

$$
\frac{I_{\alpha, \beta}^{j+1} f(z)}{z}=\frac{\beta z w^{\prime}(z)+(\alpha+\beta) w(z)}{(\alpha+\beta)} .
$$

Further computations give

$$
\frac{I_{\alpha, \beta}^{j+2} f(z)}{z}=\frac{\beta^{2} z^{2} w^{\prime \prime}(z)+\left(3 \beta^{2}+2 \alpha \beta\right) z w^{\prime}(z)+(\alpha+\beta)^{2} w(z)}{(\alpha+\beta)^{2}},
$$


and

$$
\frac{I_{\alpha, \beta}^{j+3} f(z)}{z}=\frac{\begin{array}{c}
\beta^{3} z^{3} w^{\prime \prime \prime}(z)+\left(3 \alpha \beta^{2}+6 \beta^{3}\right) z^{2} w^{\prime \prime}(z)+\left(9 \alpha \beta^{2}+3 \alpha^{2} \beta+7 \beta^{3}\right) z w^{\prime}(z)+ \\
(\alpha+\beta)^{3} w(z)
\end{array}}{(\alpha+\beta)^{3}} .
$$

Now, we will express a transformation from $\mathbb{C}^{4}$ to $\mathbb{C}$ by

$$
\begin{aligned}
& u(r, s, t, e)=r, v(r, s, t, e)=\frac{\beta s+(\alpha+\beta) r}{(\alpha+\beta)}, x(r, s, t, e)=\frac{\beta^{2} t+\left(3 \beta^{2}+2 \alpha \beta\right) s+(\alpha+\beta)^{2} r}{(\alpha+\beta)^{2}}, \\
& \text { and } y(r, s, t, e)=\frac{\beta^{3} e+\left(3 \alpha \beta^{2}+6 \beta^{3}\right) t+\left(9 \alpha^{2} \beta+3 \alpha^{2} \beta+7 \beta^{3}\right) s+(\alpha+\beta)^{3} r}{(\alpha+\beta)^{3}} .
\end{aligned}
$$

Next, suppose that

$$
\begin{array}{r}
\psi(r, s, t, e ; z)=\phi(u, v, x, y ; z)=\psi\left(r, \frac{\beta s+(\alpha+\beta) r}{(\alpha+\beta)}, \frac{\beta^{2} t+\left(3 \beta^{2}+2 \alpha \beta\right) s+(\alpha+\beta)^{2} r}{(\alpha+\beta)^{2}},\right. \\
\left.\frac{\beta^{3} e+\left(3 \alpha \beta^{2}+6 \beta^{3}\right) t+\left(9 \alpha^{2} \beta+3 \alpha^{2} \beta+7 \beta^{3}\right) s+(\alpha+\beta)^{3} r}{(\alpha+\beta)^{3}} ; z\right)
\end{array}
$$


and

$$
\phi\left(\frac{I_{\alpha, \beta}^{j} f(z)}{z}, \frac{I_{\alpha, \beta}^{j+1} f(z)}{z}, \frac{I_{\alpha, \beta}^{j+2} f(z)}{z}, \frac{I_{\alpha, \beta}^{j+3} f(z)}{z} ; z\right) \prec h(z),
$$

then

$$
\frac{I_{\alpha, \beta}^{j} f(z)}{z} \prec q(z) .
$$

Proof. It is clear that from Theorem 6, we arrive at the outcome.

The next corollaries are extensions of Theorem 6 to the case where the behavior of $q$ on $\partial U$ is not known.

Corollary 6. Let $\Omega \subset \mathbb{C}$ and suppose that $q(z)$ is univalent function in $U$ with $q(0)=1$. Let $\phi \in \Phi_{I, 2}\left[\Omega, q_{\rho}\right]$ for some $\rho \in(0,1)$, where $q_{\rho}(z)=q(\rho z)$. If $f \in \mathcal{A}(n)$ and $q_{\rho} \in Q_{0}$ fulfills

$$
\operatorname{Re}\left\{\frac{\zeta q_{\rho}^{\prime \prime}(\zeta)}{q_{\rho}^{\prime}(\zeta)}\right\} \geq 0,\left|(\alpha+\beta)\left(\frac{I_{\alpha, \beta}^{j+1} f(z)-I_{\alpha, \beta}^{j} f(z)}{z}\right)\right| \leq k \beta\left|q_{\rho}^{\prime}(\zeta)\right|,
$$

and

$$
\phi\left(\frac{I_{\alpha, \beta}^{j} f(z)}{z}, \frac{I_{\alpha, \beta}^{j+1} f(z)}{z}, \frac{I_{\alpha, \beta}^{j+2} f(z)}{z}, \frac{I_{\alpha, \beta}^{j+3} f(z)}{z} ; z\right) \in \Omega,
$$

then

$$
\begin{gathered}
\frac{I_{\alpha, \beta}^{j} f(z)}{z} \prec q(z), \\
\left(z \in U, \quad \zeta \in \partial U \backslash E\left(q_{\rho}\right), \quad \beta>0 \text { and } k \in \mathbb{N} \backslash\{1\}\right) .
\end{gathered}
$$

Proof. As a consequence of Theorem 6, that $\frac{I_{\alpha, \beta}^{j} f(z)}{z} \prec q_{\rho}(z)$.

Now, the outcome may be deduce from $q_{\rho}(z) \prec q(z)$

The proof of Corollary 6 is complete.

Corollary 7. Let $\Omega \subset \mathbb{C}$ and suppose that $q(z)$ is univalent function in $U(q(0)=1)$. Let $\phi \in \Phi_{I, 1}\left[\Omega, q_{\rho}\right]$ for some $\rho \in(0,1)$, where $q_{\rho}(z)=q(\rho z)$. If $f \in \mathcal{A}(n)$ and $q_{\rho} \in Q_{0}$ fulfills

$$
\operatorname{Re}\left\{\frac{\zeta q_{\rho}^{\prime \prime}(\zeta)}{q_{\rho}^{\prime}(\zeta)}\right\} \geq 0, \quad(\alpha+\beta)\left(\frac{I_{\alpha, \beta}^{j+1} f(z)-I_{\alpha, \beta}^{j} f(z)}{z}\right)|\leq k \beta| q_{\rho}^{\prime}(\zeta) \mid,
$$

and

$$
\phi\left(\frac{I_{\alpha, \beta}^{j} f(z)}{z}, \frac{I_{\alpha, \beta}^{j+1} f(z)}{z}, \frac{I_{\alpha, \beta}^{j+2} f(z)}{z}, \frac{I_{\alpha, \beta}^{j+3} f(z)}{z} ; z\right) \prec h(z),
$$

then

$$
\frac{I_{\alpha, \beta}^{j} f(z)}{z} \prec q(z),
$$




$$
\left(z \in U, \zeta \in \partial U \backslash E\left(q_{\rho}\right), \quad \beta>0 \text { and } k \in \mathbb{N} \backslash\{1\}\right) .
$$

Theorem 8. Assume $\phi: \mathbb{C}^{4} \times U \rightarrow \mathbb{C}, h$ is univalent in $U$ and $\psi$ is given by (9). Assume the differential equation

$$
\psi\left(q(z), z q^{\prime}(z), z^{2} q^{\prime \prime}(z), z^{3} q^{\prime \prime \prime}(z) ; z\right)=h(z)
$$

has a solution $q(z) \in Q_{1} \cap \mathcal{H}$. If the function $f \in \mathcal{A}(n)$ fulfills the condition (19) and

$$
\phi\left(\frac{I_{\alpha, \beta}^{j} f(z)}{z}, \frac{I_{\alpha, \beta}^{j+1} f(z)}{z}, \frac{I_{\alpha, \beta}^{j+2} f(z)}{z}, \frac{I_{\alpha, \beta}^{j+3} f(z)}{z} ; z\right), \beta>0
$$

is analytic in $U$, then (19) implies that

$$
\frac{I_{\alpha, \beta}^{j} f(z)}{z} \prec q(z)
$$

and $q(z)$ is the best dominant.

Proof. From Theorem 6, we find that $q(z)$ is a dominant (19) since $q(z)$ fulfills:

$$
\psi\left(q(z), z q^{\prime}(z), z^{2} q^{\prime \prime}(z), z^{3} q^{\prime \prime \prime}(z) ; z\right)=h(z),
$$

it is also a solution of the above differential equation and therefore $q(z)$ will be dominated by all dominants.

\section{Third-Order Differential Superordination Properties}

This part analyzes the third-order differential superordination properties.

Definition 9. Let $\Omega$ be a set in $\mathbb{C}, q \in \mathcal{H}$ with $q^{\prime}(z) \neq 0$ and $m \in \mathbb{N} \backslash\{1\}$. The class of admissible functions $\Phi_{I, 1}^{\prime}[\Omega, q]$ consists of those functions $\phi: \mathbb{C}^{4} \times \bar{U} \rightarrow \mathbb{C}$ which fulfill the admissibility condition

$$
\psi(u, v, x, y ; \zeta) \in \Omega,
$$

whenever

$$
\begin{gathered}
u=q(z), v=\frac{k z \beta q^{\prime}(z)+m \alpha q(z)}{m(\alpha+\beta)}, \\
\operatorname{Re}\left\{\frac{(\alpha+\beta)^{2} x-2 \alpha(v \alpha+v \beta)+\alpha^{2} u}{\beta(v(\alpha+\beta)-\alpha u)}-1\right\} \leq \frac{1}{m} \operatorname{Re}\left\{\frac{z q^{\prime \prime}(z)}{q^{\prime}(z)}+1\right\}
\end{gathered}
$$

and

$\operatorname{Re}\left\{\frac{(\alpha+\beta)^{3} y-(3 \alpha+3 \beta)(\alpha+\beta)^{2} x+9 \alpha^{2} v \beta+6 \alpha v \beta^{2}-3 \alpha^{2} u \beta-\alpha^{3} u+3 \alpha^{3} v}{\beta^{2}(v(\alpha+\beta)-\alpha u)}+2\right\} \leq \frac{1}{m^{2}} \operatorname{Re}\left\{\frac{z^{2} q^{\prime \prime \prime}(z)}{q^{\prime}(z)}\right\}$,

$$
(z \in U, \beta>0 \text { and } \zeta \in \partial U, m \in \mathbb{N} \backslash\{1\}) .
$$

Theorem 9. Let $\phi \in \Phi_{I, 1}^{\prime}[\Omega, q]$. If the functions $f \in \mathcal{A}(n), \beta>0$ and $I_{\alpha, \beta}^{j} f(z) \in Q_{0}$ fulfills the following conditions

$$
\operatorname{Re}\left\{\frac{z q^{\prime \prime}(z)}{q^{\prime}(z)}\right\} \geq 0, \quad\left|(\alpha+\beta) I_{\alpha, \beta}^{j+1} f(z)-\alpha I_{\alpha, \beta}^{j} f(z)\right| \leq m \beta\left|q^{\prime}(z)\right|
$$


and

$$
\phi\left(I_{\alpha, \beta}^{j} f(z), I_{\alpha, \beta}^{j+1} f(z), I_{\alpha, \beta}^{j+2} f(z), I_{\alpha, \beta}^{j+3} f(z) ; z\right)
$$

is univalent in $U$, then

$$
\Omega \subset\left\{\phi\left(I_{\alpha, \beta}^{j} f(z), I_{\alpha, \beta}^{j+1} f(z), I_{\alpha, \beta}^{j+2} f(z), I_{\alpha, \beta}^{j+3} f(z) ; z\right): z \in U\right\},
$$

implies that

$$
q(z) \prec I_{\alpha, \beta}^{j} f(z)
$$

Proof. Let the function $w(z)$ be given by (6) and $\psi$ be given by (9). Since $\psi \in \Phi_{I, 1}^{\prime}[\Omega, q]$, the Equations (10) and (21) imply that

$$
\Omega \subset \psi\left(w(z), z w^{\prime}(z), z^{2} w^{\prime \prime}(z), z^{3} w^{\prime \prime \prime}(z) ; z\right) .
$$

This follows easily from (9), the admissible condition for $\psi \in \Phi_{I, 1}^{\prime}[\Omega, q]$ in Definition 9 is equivalent to the admissible condition for $\psi \in \psi_{I, 1}^{\prime}[\Omega, q]$ as given in Definition 5 for $n=2$. Hence, by using the conditions in (20) and from Theorem 2, we obtain

$$
q(z) \prec w(z),
$$

or, equivalently

$$
q(z) \prec I_{\alpha, \beta}^{j} f(z) .
$$

If $\Omega \neq \mathbb{C}$ is a simply connected domain and $\Omega=h(U)$ for some conformal mapping $h$ of $U$ onto $\Omega$, then the class $\Phi_{I, 1}^{\prime}[h(U), q]$ is expressed by $\Phi_{I, 1}^{\prime}[h, q]$. Proceeding similarly as in the previous section, the following outcome is a consequence of Theorem 9.

Theorem 10. Let $\phi \in \Phi_{I, 1}^{\prime}[h, q]$ and assume $h$ is analytic in $U$. If the functions $f \in \mathcal{A}(n)$ and $I_{\alpha, \beta}^{j} f(z) \in Q_{0}$ fulfill the condition (20) and

$$
\phi\left(I_{\alpha, \beta}^{j} f(z), I_{\alpha, \beta}^{j+1} f(z), I_{\alpha, \beta}^{j+2} f(z), I_{\alpha, \beta}^{j+3} f(z) ; z\right)
$$

is univalent in $U$, then

$$
h(z) \prec \phi\left(I_{\alpha, \beta}^{j} f(z), I_{\alpha, \beta}^{j+1} f(z), I_{\alpha, \beta}^{j+2} f(z), I_{\alpha, \beta}^{j+3} f(z) ; z\right)
$$

implies that

$$
q(z) \prec I_{\alpha, \beta}^{j} f(z)
$$

Proof. The proof is deduce from Theorem 9.

Next, we will give the existence of best subordinant of (22) for a suitable $\psi$.

Theorem 11. Let $\phi: \mathbb{C}^{4} \times \bar{U} \rightarrow \mathbb{C}$ be given by (9) and $h$ be analytic in $U$. Assume the differential equation:

$$
\psi\left(q(z), z q^{\prime}(z), z^{2} q^{\prime \prime}(z), z^{3} q^{\prime \prime \prime}(z) ; z\right)=h(z),
$$

has a solution $q(z) \in Q_{0}$. If $f(z) \in \mathcal{A}(n)$ and $I_{\alpha, \beta}^{j} f(z) \in Q_{0}$ satisfy the conditions (20) and 


$$
\phi\left(I_{\alpha, \beta}^{j} f(z), I_{\alpha, \beta}^{j+1} f(z), I_{\alpha, \beta}^{j+2} f(z), I_{\alpha, \beta}^{j+3} f(z) ; z\right), \beta>0,
$$

is univalent in $U$, then (20) implies that

$$
q(z) \prec I_{\alpha, \beta}^{j} f(z)
$$

and $q(z)$ is the best subordinant.

Proof. From Theorem 9, we find that $q(z)$ is a subordinant (22) since $q(z)$ fulfills

$$
\psi\left(q(z), z q^{\prime}(z), z^{2} q^{\prime \prime}(z), z^{3} q^{\prime \prime \prime}(z) ; z\right)=h(z),
$$

it is also a solution of the above differential equation and therefore $q(z)$ will be subordinated by all subordinants.

The following sandwich-type result is obtained by combining Theorem 4 and Theorem 10 .

Theorem 12. Let the functions $h_{1}, q_{1}$ be analytic in $U, h_{2}$ be univalent in $U, q_{2}(z) \in Q_{0}$ with $q_{1}(0)=q_{2}(0)=1$ and $\phi \in \Phi_{I, 1}\left[, h_{2}, q_{2}\right] \cap \Phi_{I, 1}^{\prime}\left[h_{1}, q_{1}\right]$. If the functions $f \in \mathcal{A}(n)$, $I_{\alpha, \beta}^{j} f(z) \in Q_{0} \cap \mathcal{H}$ and $\phi\left(I_{\alpha, \beta}^{j} f(z), I_{\alpha, \beta}^{j+1} f(z), I_{\alpha, \beta}^{j+2} f(z), I_{\alpha, \beta}^{j+3} f(z) ; z\right)$ is univalent in $U$, the conditions (4), (20) are satisfied, then

$$
h_{1}(z) \prec \phi\left(I_{\alpha, \beta}^{j} f(z), I_{\alpha, \beta}^{j+1} f(z), I_{\alpha, \beta}^{j+2} f(z), I_{\alpha, \beta}^{j+3} f(z) ; z\right) \prec h_{2}(z),
$$

gives that

$$
q_{1}(z) \prec I_{\alpha, \beta}^{j} f(z) \prec q_{2}(z)
$$

Proof. The result follows from Theorem 4 and Theorem 10, respectively.

Next, we establish a new admissible class $\Phi_{\mathrm{I}, 2}^{\prime}[\Omega, q]$ below.

Definition 10. Let $\Omega$ be a set in $\mathbb{C}$ and $q \in \mathcal{H}\left(q^{\prime}(z) \neq 0\right)$. The admissible functions class $\Phi_{I, 2}^{\prime}[\Omega, q]$ consists of those functions $\phi: \mathbb{C}^{4} \times \bar{U} \rightarrow \mathbb{C}$ which fulfill the admissibility condition

$$
\psi(u, v, x, y ; \zeta) \in \Omega
$$

whenever

$$
\begin{gathered}
u=q(z), \quad v=\frac{z \beta q^{\prime}(z)+m(\alpha+\beta) q(z)}{m(\alpha+\beta)} \\
\operatorname{Re}\left\{\frac{(\alpha+\beta)^{2} x-\beta^{2} u+\alpha^{2}(u-2 v)-2 \alpha \beta v}{\beta((\alpha+\beta) v-(\alpha+\beta) u)}-2\right\} \leq \frac{1}{m} \operatorname{Re}\left\{\frac{z q^{\prime \prime}(z)}{q^{\prime}(z)}+1\right\}
\end{gathered}
$$

and

$\operatorname{Re}\left\{\frac{(\alpha+\beta)^{3} y-(3 \alpha+6 \beta)(\alpha+\beta)^{2} x+15 \beta \alpha^{2} v+12 \alpha \beta^{2} v+3 \alpha^{3} v-\alpha^{3} u-6 \alpha \beta u^{3}+5 \beta^{3} u}{\beta^{2}(v(\alpha+\beta)-(\alpha+\beta) u)}+11\right\} \leq \frac{1}{m^{2}} \operatorname{Re}\left\{\frac{z^{2} q^{\prime \prime \prime}(z)}{q^{\prime}(z)}\right\}$,

$$
(z \in U, \beta>0 \text { and } \zeta \in \partial U, m \in \mathbb{N} \backslash\{1\}) \text {. }
$$

Theorem 13. Let $\psi \in \Phi_{I, 2}^{\prime}[\Omega, q]$. If the functions $f \in \mathcal{A}(n), \frac{{ }_{\alpha, \beta}^{j} f(z)}{z} \in Q_{1}$ and $q \in \mathcal{H}$ $\left(q^{\prime}(z) \neq 0\right)$ fulfill the following conditions: 


$$
\begin{gathered}
\operatorname{Re}\left\{\frac{\zeta q^{\prime \prime}(\zeta)}{q^{\prime}(\zeta)}\right\} \geq 0,\left|(\alpha+\beta)\left(\frac{I_{\alpha, \beta}^{j+1} f(z)-I_{\alpha, \beta}^{j} f(z)}{z}\right)\right| \leq m \beta\left|q^{\prime}(\zeta)\right|, \quad \beta>0 \\
\phi\left(\frac{I_{\alpha, \beta}^{j} f(z)}{z}, \frac{I_{\alpha, \beta}^{j+1} f(z)}{z}, \frac{I_{\alpha, \beta}^{j+2} f(z)}{z}, \frac{I_{\alpha, \beta}^{j+3} f(z)}{z} ; z\right)
\end{gathered}
$$

is univalent in $U$, then

$$
\Omega \subset\left\{\phi\left(\frac{I_{\alpha, \beta}^{j} f(z)}{z}, \frac{I_{\alpha, \beta}^{j+1} f(z)}{z}, \frac{I_{\alpha, \beta}^{j+2} f(z)}{z}, \frac{I_{\alpha, \beta}^{j+3} f(z)}{z} ; z\right): z \in U\right\},
$$

implies

$$
q(z) \prec \frac{I_{\alpha, \beta}^{j} f(z)}{z} .
$$

Proof. Let the function $w(z)$ be given by (14) and $\psi$ be defined by (17). Since $\psi \in \Phi_{I, 2}^{\prime}[\Omega, q]$, the Equations (24) and (18) imply

$$
\Omega \subset \psi\left(w(z), z w^{\prime}(z), z^{2} w^{\prime \prime}(z), z^{3} w^{\prime \prime \prime}(z) ; z\right) .
$$

This follows easily from (17) that the admissible condition for $\psi \in \Phi_{I, 2}^{\prime}[\Omega, q]$ in Definition 10 is equivalent to the admissible condition for $\psi \in \Psi_{I, 2}^{\prime}[\Omega, q]$ as given in Definition 5 for $n=2$. Hence, by using the conditions in (23) and applying Theorem 2, we find

$$
q(z) \prec w(z) .
$$

If $\Omega \neq \mathbb{C}$ is a simply connected domain and $\Omega=h(U)$ for some conformal mapping $h$ of $U$ on to $\Omega$, then the class $\psi \in \Phi_{I, 2}^{\prime}[h(U), q]$ is expressed by $\psi \in \Phi_{I, 2}^{\prime}[h, q]$.

Theorem 14. Let $\psi \in \Phi_{I, 2}^{\prime}[h, q]$ and $h$ be analytic in $U$. If the functions $f \in \mathcal{A}(n)$ and $\frac{I_{\alpha, \beta}^{j} f(z)}{z} \in Q_{1}$ fulfills the condition (23) and

$$
\phi\left(\frac{I_{\alpha, \beta}^{j} f(z)}{z}, \frac{I_{\alpha, \beta}^{j+1} f(z)}{z}, \frac{I_{\alpha, \beta}^{j+2} f(z)}{z}, \frac{I_{\alpha, \beta}^{j+3} f(z)}{z} ; z\right)
$$

is univalent in $U$, then

$$
h(z) \prec \phi\left(\frac{I_{\alpha, \beta}^{j} f(z)}{z}, \frac{I_{\alpha, \beta}^{j+1} f(z)}{z}, \frac{I_{\alpha, \beta}^{j+2} f(z)}{z}, \frac{I_{\alpha, \beta}^{j+3} f(z)}{z} ; z\right),
$$

implies

$$
q(z) \prec \frac{I_{\alpha, \beta}^{j} f(z)}{z}, \beta>0 .
$$

Proof. It is clear that by using Theorem 13, we find the desired outcome.

Theorem 15. Let $\phi: \mathbb{C}^{4} \times \bar{U} \rightarrow \mathbb{C}$, the function $h$ be analytic in $U$ and $\psi$ be defined by (17). Assume that the differential equation 


$$
\psi\left(q(z), z q^{\prime}(z), z^{2} q^{\prime \prime}(z), z^{3} q^{\prime \prime \prime}(z) ; z\right)=h(z)
$$

has a solution $q(z) \in Q_{1}$. If $f \in \mathcal{A}(n)$ and $\frac{I_{\alpha, \beta}^{j} f(z)}{z} \in Q_{1}$ fulfills the condition (23) and

$$
\phi\left(\frac{I_{\alpha, \beta}^{j} f(z)}{z}, \frac{I_{\alpha, \beta}^{j+1} f(z)}{z}, \frac{I_{\alpha, \beta}^{j+2} f(z)}{z}, \frac{I_{\alpha, \beta}^{j+3} f(z)}{z} ; z\right)
$$

is univalent in $U$, then (25) gives that

$$
q(z) \prec \frac{I_{\alpha, \beta}^{j} f(z)}{z},
$$

and $q$ is the best subordinant.

Proof. The proof is similar to that of Theorem 8 .

The next sandwich-type outcome is obtained by combining Theorem 7 and Theorem 14 .

Theorem 16. Let the functions $h_{1}, q_{1}$ be analytic in $U, h_{2}$ be univalent in $U$, $q_{2} \in Q_{1}\left(q_{1}(0)=q_{2}(0)=1\right)$ and $\phi \in \Phi_{I, 2}\left[h_{2}, q_{2}\right] \cap \Phi_{I, 2}^{\prime}\left[h_{1}, q_{1}\right]$. If $f(z) \in \mathcal{A}(n)$, $\frac{I_{\alpha, \beta}^{j} f(z)}{z} \in Q_{1} \cap \mathcal{H}$ and

$$
\psi\left(\frac{I_{\alpha, \beta}^{j} f(z)}{z}, \frac{I_{\alpha, \beta}^{j+1} f(z)}{z}, \frac{I_{\alpha, \beta}^{j+2} f(z)}{z}, \frac{I_{\alpha, \beta}^{j+3} f(z)}{z} ; z\right)
$$

is univalent in $U$, the conditions (12), (23) are satisfied, then

$$
h_{1}(z) \prec \phi\left(\frac{I_{\alpha, \beta}^{j} f(z)}{z}, \frac{I_{\alpha, \beta}^{j+1} f(z)}{z}, \frac{I_{\alpha, \beta}^{j+2} f(z)}{z}, \frac{I_{\alpha, \beta}^{j+3} f(z)}{z} ; z\right) \prec h_{2}(z),
$$

implies that

$$
q_{1}(z) \prec \frac{I_{\alpha, \beta}^{j} f(z)}{z} \prec q_{2}(z)
$$

\section{Conclusions and Future Work}

We aim to give some outcomes for third-order differential subordination and superordination for analytic functions in $U=\{z \in \mathbb{C}:|z|<1\}$ involving the generalized operator $I_{\alpha, \beta}^{j} f$. The outcomes are derived by investigating relevant classes of admissible functions. Some new outcomes on differential subordination and superordination with some sandwich theorems are expressed. Moreover, several particular cases are also noted. The properties and outcomes of the differential subordination are symmetry to the properties of the differential superordination to form the sandwich theorems. The outcomes included in this current paper reveal new ideas for continuing the study, and we open some windows for researchers to generalize the classes to establish new outcomes in univalent and multivalent function theory.

Author Contributions: Conceptualization, methodology, software, validation, formal analysis, investigation, resources, by S.A., data curation, writing —original draft preparation, writing-review and editing, visualization by R.A.H., supervision, project administration, funding acquisition, by W.G.A. All authors have read and agreed to the published version of the manuscript. 
Funding: This research received no external funding.

Institutional Review Board Statement: Not applicable.

Informed Consent Statement: Not applicable.

Data Availability Statement: Not applicable.

Conflicts of Interest: The authors declare no conflict of interest.

\section{References}

1. Miller, S.S.; Mocanu, P.T. Differential Subordination: Theory and Applications; Series on Monographs and Textbooks in Pure and Applied Mathematics; Marcel Dekker Incorporated: New York, NY, USA, 2000; Volume 225.

2. Swamy, S.R. Inclusion Properties of Certain Subclasses of Analytic Functions. Int. Math. Forum 2012, 7, 1751-1760.

3. Al-Oboudi, F.M. On Univalent Functions Defined by a Generalized Salagean Operator. Int. J. Math. Math. Sci. 2004, 27, 1429-1436. [CrossRef]

4. Cho, N.E.; Srivastava, H.M. Argument Estimates of Certain Analytic Functions Defined by a Class of Multiplier Transformations. Math. Comput. Model. 2003, 37, 39-49. [CrossRef]

5. Cho, N.E.; Kim, T.H. Multiplier Transformations and Strongly Close-to-Convex Functions. Bull. Korean Math. Soc. 2003, 40, 399-410. [CrossRef]

6. Catas, A. On Certain Classes of p-Valent Functions Defined by Multiplier Transformations. In Proceedings of the International Symposium on Geometric Function Theory and Applications: GFTA 2007 Proceedings, Istanbul, Turkey, 20-24 August 2007; Owa, S., Polatog,lu, Y., Eds.; TC İstanbul Kültür University Publications. TC İstanbul Kültür University: İstanbul, Turkey, 2008; Volume 91, pp. 241-250.

7. Antonino, J.A.; Miller, S.S. Third-Order Differential Inequalities and Subordinations in the Complex Plane. Complex Var. Elliptic Equ. 2011, 56, 439-454. [CrossRef]

8. Tang, H.; Deniz, E. Third-Order Differential Subordination Results for Analytic Functions Involving the Generalized Bessel Functions. Acta Math. Sci. 2014, 34, 1707-1719. [CrossRef]

9. Tang, H.; Srivastava, H.M.; Deniz, E.; Li, S.-H.; Ma, L. Third-Order Differential Superordination Involving the Generalized Bessel Functions. Bull. Malays. Math. Sci. Soc. 2015, 38, 1669-1688. [CrossRef]

10. Miller, S.S.; Mocanu, P.T. Subordinants of Differential Superordinations. Complex Var. Theory Appl. Int. J. 2003, 48, 815-826. [CrossRef]

11. Miller, S.S.; Mocanu, P.T.; Reade, M.O. Subordination-preserving integral operators. Trans. Amer. Math. Soc. 1984, $283,605-615$. [CrossRef]

12. Al-Ameedee, S.A.; Atshan, W.G.; Al-Maamori, F.A. On Sandwich Results of Univalent Functions Defined by a Linear Operator. J. Interdiscip. Math. 2020, 23, 803-809. [CrossRef]

13. Atshan, W.G.; Ali, A.A.R. On Some Sandwich Theorems of Analytic Functions Involving Noor-Sălăgean Operator. Adv. Math. Sci. J. 2020, 9, 8455-8467. [CrossRef]

14. Atshan, W.G.; Hassan, H.Z.; Yalcin, S. On Third-Order Differential Subordination Results for Univalent Functions Defined by Differential Operator. Uzb. Math. J. 2021, 65, 26-42.

15. Agarwal, P.; Hyder, A.A.; Zakarya, M. Well-Posedness of Stochastic Modified Kawahara Equation. Adv. Differ. Equ. 2020, 1, 1-10. [CrossRef]

16. AlNemer, G.; Zakarya, M.; El-Hamid, H.A.A.; Kenawy, M.R.; Rezk, H.M. Dynamic Hardy-Type Inequalities with Non-Conjugate Parameters. Alex. Eng. J. 2020, 59, 4523-4532. [CrossRef]

17. Rezk, H.M.; AlNemer, G.; Abd El-Hamid, H.A.; Abdel-Aty, A.H.; Nisar, K.S.; Zakarya, M. Hilbert-Type Inequalities for Time Scale Nabla Calculus. Adv. Differ. Equ. 2020, 1, 1-21. [CrossRef]

18. Ali, R.M.; Ravichandran, V.; Seenivasagan, N. Subordination and Superordination of the Liu-Srivastava Linear Operator on Meromorphic Functions. Bull. Malays. Math. Sci. Soc. 2008, 31, 193-207.

19. Darweesh, A.M.; Atshan, W.G.; Battor, A.H.; Lupas, A.A. Third-Order Differential Subordination Results for Analytic Functions Associated with a Certain Differential Operator. Symmetry 2022, 14, 99. [CrossRef]

20. Rahman, I.A.R.; Atshan, W.G.; Oros, G.I. New Concept on Fourth Hankel Determinant of a Certain Subclass of Analytic Functions. Afrika Matematika 2022, 33, 7. [CrossRef] 